\title{
Revista Leterar Pacar
}

ISSN: 2317-2347 - v. 9, Dossier Spécial (2020)

Todo o conteúdo da RLR está licenciado sob Creative Commons Atribuição 4.0 Internacional

\section{De l'imitation à la création en langue étrangère: pouvoir être}

\section{un autre/ Da imitação à criação em língua estrangeira: poder ser}

\section{um outro}

\section{Cristina Moerbeck Casadei Pietraroia *}

Titulaire d'une licence en français et en portugais par l'Université de São Paulo (1986), d'une maîtrise en Sciences du Langage, obtenue à l'Université Stendhal Grenoble 3, (1990) et d'un doctorat en Lettres (langue et littérature françaises) à l'Université de São Paulo (1996). Maître de conférences et chercheuse dans le domaine des études linguistiques, littéraires et de la traduction en français à la Faculté de philosophie, lettres et sciences humaines de l'Université de São Paulo. Elle a reçu le prix F de la Francophonie, décerné par le journal Le Franc-Parler, et le titre de Chevalier des Palmes Académiques, par le gouvernement français. Elle a été présidente de l'Association des professeurs de français de l'État de São Paulo et a travaillé comme professeur invité au Département des Langues Romanes de l'Université Lumière Lyon 2.

iD https://orcid.org/0000-0001-8110-1767

Reçu en 11 nov. 2020. Approuvé en: 23 nov. 2020.

\section{Comment citer cet article:}

PIETRAROIA, Cristina Casadei. De l'imitation à la création en langue étrangère: pouvoir être un autre. Revista Letras Raras. Campina Grande, v. 9, p. 12-32, nov. 2020.

\section{RÉSUMÉ}

Cet article aborde le passage obligatoire, dans l'apprentissage d'une langue étrangère, par le plaisir, le jeu, l'imitation et la création. Le contexte est celui de l'enseignement du français langue étrangère au niveau de la Licence dans une université brésilienne où les étudiants, dont la plupart sont débutants, doivent non seulement apprendre cette langue mais aussi en faire un objet de travail. II faut donc qu'ils la maîtrisent bien et, pour cela, ils doivent être préparés à mettre en question leurs propres identités et à se montrer dans ce qu'ils ont de plus subjectif : leurs souvenirs et leurs émotions.

MOTS-CLES : Enseignement-apprentissage; Français Langue Étrangère ; Jeux dramatiques ; Subjectivité ; Identité.

\section{RESUMO}

Este artigo aborda a passagem obrigatória, durante a aprendizagem de uma língua estrangeira, pelo prazer, pelos jogos, pela imitação e pela criação. O contexto de pesquisa apresentado é o do ensino de Francês Língua Estrangeira na graduação de uma universidade brasileira onde os estudantes, sendo em sua maioria principiantes, devem não apenas aprender essa língua mas também torná-la seu objeto de trabalho. É preciso, portanto, que eles a conheçam profundamente e, para isso, devem estar preparados para questionar suas próprias identidades e se mostrar naquilo que têm de mais subjetivo: suas lembranças e emoções.

PALAVRAS-CHAVES: Ensino-aprendizagem; Francês Língua Estrangeira; Jogos teatrais; Subjetividade ; Identidade. 


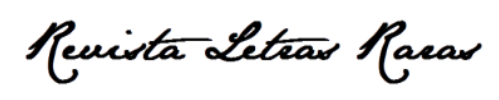

ISSN: 2317-2347 - v. 9, Dossier Spécial (2020)

Todo o conteúdo da RLR está licenciado sob Creative Commons Atribuição 4.0 Internacional

\section{Introduction}

C'est souvent l'écoute qui nous touche, nous enchante ou nous agace : des sons nouveaux, qu'on n'arrive pas à découper et dont les sens nous échappent entièrement, sont perçus avec autant de fascination que de rejet ou de peur. Nous leur attribuons tout de suite des qualificatifs beaux, bizarres, chantants - choisis aussi en fonction de nos sentiments envers ceux qui les utilisent : la douceur de la langue parlée par une grand-mère bienveillante d'origine étrangère ; la fascination ressentie par une mère qui voit son enfant parler une autre langue ; la curiosité suscitée par un parler tonal entendu pour la première fois et émanant d'une toute petite personne aux traits orientaux et aux gestes délicats ; l'enchantement de la petite fille qui écoute son père étudier les leçons d'une nouvelle langue ; la chanson inconnue jouée à la radio et qui donne envie de danser et d'aimer ; la peur des sons émis par des étrangers dont on vient de connaître l'existence, qui semblent égratigner nos oreilles et que notre ignorance rejette comme trop différents ... Ainsi, pour un Brésilien, le français paraît-il en général, très beau et élégant ; l'italien, coloré, vibrant; l'arabe et le yiddish, complètement opaques et mystérieux... En fin de compte, il nous est impossible d'écouter une langue étrangère et d'y rester indifférents, sans émotion.

La curiosité et la fascination amènent les personnes à vouloir apprendre des langues étrangères avec ce qu'elles ont à leur portée, de façon autodidacte ou dans un contexte d'enseignement (une école de langue, des cours privés), parfois à des milliers de kilomètres de distance des pays où ces langues sont parlées. Cette situation d'apprentissage qu'on nomme " exolingue ${ }^{1}$ peut être aussi imposée à un apprenant ${ }^{2}$, à des âges et à des moments variés de sa vie, ce qui n'exclut pas l'amour, la haine ou des sentiments moins intenses par rapport à la nouvelle langue. II suffit d'écouter les témoignages d'adultes à propos de l'enseignant de français ou d'anglais qu'ils aimaient ${ }^{3}$, grâce à qui ils ont continué à étudier la langue en question ou, au contraire, à cause de qui ils ont été incapables de l'apprendre « pour toujours »!

\footnotetext{
1 Les adjectifs « exolingue » et " endolingue » permettent de distinguer deux situations d'enseignement d'une langue étrangère. Ils sont ainsi définis par Louise Dabène et al. $(1990$, p. 9) : contexte exolingue «le cas où il est dispensé dans un pays où l'on parle une autre langue enseignée, celle-ci pouvant être ou non la langue maternelle des apprenants »; contexte endolingue : «le cas où cet enseignement est dispensé dans un pays où l'on parle la langue enseignée. D'autres auteurs affirment que ce sont les situations de communication qui peuvent être endolingues ou exolingues, mais nous gardons, dans cet article, la définition de Dabène et al. (1990, p. 9).

2 Les termes " apprenant » et " enseignant » font référence au féminin et au masculin.

3 Le cas de l'enseignante de français Iracema Rosa dos Santos, appelée " Dona Boquinha » par ses élèves, décrit par Marcella dos Santos Abreu (2019) dans sa thèse de doctorat est très illustratif de cette question. Un autre cas, tout aussi intéressant, est celui des souvenirs de H.F., décrit par Sahsha Dellatorre (2012) dans son mémoire de Master.
} 


\section{Revista Leterar Pacar}

ISSN: 2317-2347 - v. 9, Dossier Spécial (2020)

Todo o conteúdo da RLR está licenciado sob Creative Commons Atribuição 4.0 Internacional

Dans le cadre de ce texte, ce qui nous intéresse est la situation d'enseignementapprentissage du Français Langue Etrangère (FLE), au niveau de la Licence, dans une université brésilienne. Les étudiants qui choisissent cette filière sont âgés de 18 à 30 ans en moyenne, et comptent non seulement apprendre le français mais aussi être formés pour l'enseigner, le traduire, enfin, l'utiliser dans leur vie professionnelle. Avant, pourtant, d'aborder ce contexte, nous nous proposons d'analyser d'autres situations d'enseignement-apprentissage du français dans leur complexité.

\section{Des passions et des conflits envers les langues}

Vouloir apprendre une langue n'exclut pas, loin de là, les difficultés et les souffrances qui marquent tout apprentissage, comme en témoigne François Cheng dans un extrait de son livre Le dialogue (2002) :

Débarquant à Paris à dix-neuf ans passés, je ne connaissais pas un mot de français. Pas plus que je n'étais en état de mesurer toute la difficulté qui m'attendait à entrer, à cet âge dit "tardif», dans une autre langue, à en maîtriser l'usage, à en faire ma chair et mon sang. Pour que, par la suite, j'aie osé rêver de devenir un jour écrivain français et qu'un jour effectivement je le sois devenu, il m'aura fallu de la détermination certes, mais surtout une bonne dose d'inconscience, sinon d'extravagance. Et de la patience. Un demi-siècle de tâtonnements, de perditions, de relèvements, de fulgurantes joies mêlées de larmes, d'indicibles ravissements toujours sur fond d'inquiétude, de tremblement...(CHENG, 2002, pp. 27, 28)

La poètesse brésilienne Sandra Catrouxo, à son tour, écrit sur son sentiment par rapport à la langue française, apprise à l'âge adulte, et dans laquelle elle a pu exprimer, malgré ses difficultés, des sentiments et des expériences de façon poétique, sans passer par le portugais:

Je suis une écrivaine handicapée

Je m'aventure dans des rues inconnues

Je me sens toute seule et parfois sans aide

Je suis une écrivaine handicapée

Perdue, abandonnée

Sur une route difficile

Un chemin solitaire [...] (CATROUXO, 2019, pp. 12, 13) 


\section{Revista Leterar Pacar}

ISSN: 2317-2347 - v. 9, Dossier Spécial (2020)

Todo o conteúdo da RLR está licenciado sob Creative Commons Atribuição 4.0 Internacional

En général, c'est dans l'obligation de parler une langue - cas des immigrants et des exilés - que l'on connaît le plus de souffrances. Maryam Madjidi, écrivaine d'origine iranienne et arrivée en France à l'âge de six ans, décrit ainsi le processus ardu de maîtrise du français :

Quelques semaines ont passé. La petite fille ne parle toujours pas à ses camarades. Elle ferme obstinément la bouche. Bouche scellée mais yeux et oreilles grands ouverts. Elle prend, elle enregistre, elle digère tout ce qu'elle voit et entend. Mais elle ne parle pas.

Pourtant, elle a très bien appris cette langue puisqu'elle pense déjà en français dans sa tête et imagine des dialogues où elle se défend et prouve à tous qu'elle la parle très bien.

Les autres enfants de l'école la regardent avec un air de fausse compassion mêlée de moquerie, elle est l'étrangère, celle qui ne parle pas un mot de français, la muette, la martienne, la pauvre. (MADJIDI, 2017, p. 83)

(...) À l'école, un enfant m'a dit « cochon». Je ne sais pas ce que ce mot veut dire. J'ai senti une certaine moquerie dans la voix. J'en parle à mes parents. Ils ne savent pas non plus le sens de ce mot. Nous prenons un dictionnaire français-persan. Mon père éclate de rire, il nous donne la traduction. Ma mère se met à rire aussi. Mais moi, ça ne m'amuse pas du tout. Je me suis fait traiter de cochon et je n'ai même pas compris l'insulte et ce devant tous les autres enfants de l'école . (MADJIDI, 2017, p. 84)

Madjidi nous montre, effectivement, que l'expérience endolingue, c'est-à-dire celle vécue dans le pays où l'on parle la langue étrangère étudiée, peut être extrêmement forte et dérangeante, surtout parce que l'étranger, en général, ne veut pas se faire remarquer.

Source de souffrances, la langue étrangère, adoptée ou imposée, peut être aussi source de plaisir, de bonheur et de liberté:

Ma langue maternelle, le persan, m'impose des tabous, des interdits. La
maternelle dit l'intime, c'est elle qui nous apprend la vie, l'amour, la souff
elle qui nous ouvre au monde. C'est aussi la langue de l'autocensure. Ne
ce que le mot « maternel » : il crée trop de liens. Adopter une autre lan
français, c'est choisir la liberté. On ne se marie pas avec sa mère !
français, j'étais libéré de tonnes de contraintes affectives (RAHIMI in LAV
2008, p. 24).
[...] Dans mes rêves je peux tout faire
Dans la langue de Molière
Ma plume danse et m'emporte au loin [...] (CATROUXO, 2019, p. 13)

Cependant, au fur et à mesure que l'on maîtrise de mieux en mieux la nouvelle langue, d'autres sentiments apparaissent, dont la peur de perdre sa langue maternelle et ainsi sa patrie, 


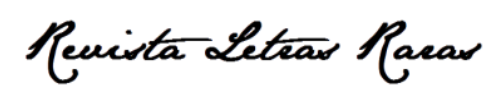

ISSN: 2317-2347 - v. 9, Dossier Spécial (2020)

Todo o conteúdo da RLR está licenciado sob Creative Commons Atribuição 4.0 Internacional

comme le décrivent Emil Cioran (« On n'habite pas un pays, on habite une langue. Une patrie, c'est cela et rien d'autre », CIORAN, 1987, p. 16), Madjidi, Cheng et Akira Mizubayashi :

Où est passé le persan ? se demandait-elle.

Elle pensait au début que la langue lui jouait un tour. Le persan s'était déguisé en français pour un temps et bientôt il réapparaîtrait à nouveau. Puis elle se dit que le persan n'avait peut-être jamais existé, que c'était un rêve. Ensuite, elle sombra dans une profonde mélancolie en pensant que le persan était mort, comme meurent les personnes, les animaux, les végétaux, comme tout ce qui vit sur cette terre. Une langue peut donc mourir? (MADJIDI, 2017, pp. 150, 151)

C'est dire que, durant au moins deux décennies après mon arrivée en France, ma vie a été marquée par un drame passionnel fait avant tout de contradictions et de déchirements. Ceux-ci, toutefois, se sont transmués peu à peu en une quête non moins passionnelle lorsque j'ai opté finalement pour une des deux langues, l'adoptant comme outil de création, sans que pour autant l'autre, celle dite maternelle, soit effacée purement et simplement. (CHENG, 2002, pp. 7, 8)

Le jour où je me suis emparé de la langue française, j'ai en effet perdu le japonais pour toujours dans sa pureté originelle. Ma langue d'origine a perdu son statut de langue d'origine. J'ai appris à parler comme un étranger dans ma propre langue. (MIZUBAYASHI, 2011, p. 261)

La question qui s'ensuit est celle posée par l'écrivain bilingue Julien Green dans Le langage et son double:

Est-on le même en français et en anglais ? Dit-on les mêmes choses ? Penset-on de la même manière dans les deux langues et avec des mots pour ainsi dire interchangeables ? Voilà des problèmes que je n'ai pas la prétention de résoudre, mais qu'il serait curieux d'examiner un peu. Souvent je suis tenté de croire que les racines du langage plongent jusqu'au profond de notre personnalité et que c'est notre façon d'être qui est en jeu quand on nous apprend à parler dans une langue ou dans une autre. Un petit [F]rançais n'appréhende pas l'univers comme le fait un petit [A]méricain, et c'est en partie à cause de la langue à travers laquelle, si l'on peut dire, cet univers leur est présenté. Après tout, nous sommes ce que nous pensons. (GREEN, 1987, p. 213)

Ces extraits sont l'exemple de l'importante complexité qui caractérise l'apprentissage et la maîtrise d'une langue étrangère, ce qu'a si bien compris et expliqué Christine Revuz dans l'un des plus beaux et meilleurs textes écrits sur la question, La langue étrangère entre le désir d'un ailleurs et le risque de l'exil :

Objet de connaissance intellectuelle, elle [la langue étrangère] est aussi objet d'une pratique. Cette pratique est elle-même complexe. Pratique d'expression, plus ou moins créative, elle sollicite le sujet, son mode de relation aux autres et au monde, pratique corporelle, elle met en jeu tout l'appareil phonatoire. [...] 


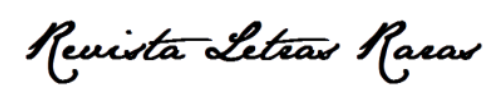

ISSN: 2317-2347 - v. 9, Dossier Spécial (2020)

Todo o conteúdo da RLR está licenciado sob Creative Commons Atribuição 4.0 Internacional

cet apprentissage [d'une langue étrangère] mobilise en effet, dans une interaction nécessaire, des dimensions de la personne qui ne sont pas nécessairement habituées à collaborer ni même à vivre en harmonie. Le sujet doit mettre au service de l'expression de son je un va-et-vient qui requiert beaucoup de souplesse psychique entre un travail du corps sur les rythmes, les sons, les courbes intonatives, et un travail d'analyse et de mémorisation des structures linguistiques. On peut faire l'hypothèse que beaucoup d'échecs peuvent s'analyser comme une incapacité à lier ces trois dimensions: affirmation du je, travail du corps, dimension cognitive. (REVUZ, 1991 pp. 25, 26. En gras dans l'original)

L'affirmation de Revuz selon laquelle «[la langue] sollicite le sujet » nous renvoie au fait que l'être humain et le langage n'en font qu'un, comme nous l'ont appris Vygotsky (2009[1934]) et d'autres spécialistes, et que cette unité se construit dans l'interaction, dans les échanges langagiers que nous entretenons avec ceux qui nous entourent dès notre vie utérine. Dans L'intime étrangeté de la langue, Irène Fenoglio s'exprime :

[l]a langue colle à nous-mêmes, nous habille et nous enrobe, nous n'avons pas pu l'éviter, nous ne pouvons pas l'éviter, mais elle nous confronte à l'étrange, à l'Autre par tout autre possible.

Intimité, complicité, altérité, étrangeté se recomposent indéfiniment, en chaque sujet, à tout instant. La langue est connivente et complice de cette infinie recherche.

Familière et maternante, étrangère et frustrante : étrange intimité de la langue. Ce sont les mots - et les mots sont de langue - qui ancrent au plus près de nous-mêmes l'incontournable étrangeté de notre propre humanité [...]. (FENOGLIO, 2006, p. 25)

La présence constitutive de l'autre, comme l'a bien montré Bakhtine (1977[1929]), résulte de nos relations toujours dialogiques, établies dans des communautés parlantes et culturellement marquées. Encore une fois, c'est Revuz (1991) qui apporte un éclairage intéressant:

Dès son premier instant de vie, il [l'enfant] est confronté à un entourage qui lui prodigue des soins et des paroles. Chacun s'affairant autour du bébé parle de lui, met en mots ce qu'il perçoit de lui, de son "caractère", de ses ressemblances, de ses besoins, et cette parole est à tout instant interprétation de ce qu'est ou ressent le bébé, prédication sur ce qu'il sera, ce que l'on attend de lui, et nomination des sensations, des affects, des objets du monde. La découverte des mots, des significations linguistiques est indissociable de l'expérience de la relation à l'autre et des significations libidinales qui s'y inscrivent. La voix, les paroles de la mère sont sources de plaisir ou de déplaisir, elles ont de surcroît le pouvoir d'interférer sur les autres sensations (vue, toucher, goût) en renforçant ou en annulant les affects de plaisir ou de déplaisir qui leur sont attachés.

Ainsi, longtemps avant de pouvoir parler, l'enfant est-il parlé intensément par son entourage, et il n'est pas un mot qui ne soit à la fois désignation d'un 


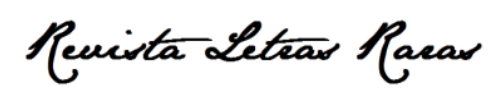

ISSN: 2317-2347 - v. 9, Dossier Spécial (2020)

Todo o conteúdo da RLR está licenciado sob Creative Commons Atribuição 4.0 Internacional

concept [au sens saussurien de signifié. Note de l'auteure] et discours sur la valeur attribuée à ce concept par l'entourage. Ce système de valeurs infiltre complètement le système linguistique. II dit ce qui peut se dire et ce qui ne saurait l'être ; il manifeste un rapport à la langue elle-même et au savoir qu'elle permet de construire.

[...] En ouvrant un nouvel espace potentiel à l'expression du sujet, la langue étrangère vient interroger la relation qui s'est instaurée entre le sujet et sa langue. Cette relation est complexe, elle est structurante du rapport que le sujet entretient avec lui-même, avec les autres, avec le savoir. (REVUZ, 1991, p. 27. En gras dans l'original.)

Les personnes qui parlent couramment une autre langue connaissent bien la situation mentionnée ci-dessus. Nancy Huston, écrivaine née au Canada anglophone et vivant en France depuis une cinquantaine d'années, affirme dans Nord perdu (1999) qu'il y a deux types de personnes bilingues : les vrais et les faux bilingues. Elle y consacre des pages entières avec une clarté étonnante :

L'acquisition d'une deuxième langue annule le caractère " naturel» de la langue d'origine - et à partir de là, plus rien n'est donné d'office, ni dans l'une ni dans l'autre ; plus rien ne vous appartient d'origine, de droit et d'évidence. (HUSTON, 1999, p. 43)

[...] Depuis longtemps, je rêve, pense, fais l'amour, écris, fantasme et pleure dans les deux langues [anglais et français] tout à tour, et parfois dans un mélange ahurissant des deux. Pourtant, elles sont loin d'occuper dans mon esprit des places comparables : comme tous les faux bilingues sans doute, j'ai souvent l'impression qu'elles font chambre à part dans mon cerveau. Loin d'être sagement couchées face à face ou dos à dos ou côte à côte, loin d'être superposées ou interchangeables, elles sont distinctes, hiérarchisées : d'abord l'une ensuite l'autre dans ma vie, d'abord l'autre ensuite l'une dans mon travail. Les mots le disent bien : la première langue, la « maternelle », acquise dès la prime enfance, vous enveloppe et vous fait sienne, alors que pour la deuxième, " adoptive ", c'est vous qui devez la materner, la maîtriser, vous l'approprier.(HUSTON, 1999, pp. 60 et 61)

On peut croire que ce ne sont que les faux bilingues qui souffrent. Or, un chapitre du livre Itinerãças, de Béatrice Reichen V. Costa, nous montre bien qu'il s'agit-là d'un leurre :

La difficulté apparaît dès le choix de la langue... Dois-je écrire en portugais ou en français ? Quelle est ma langue maternelle ? Quelle est ma première langue?

Si je me réfère au sens littéral de l'expression « langue maternelle », je peux affirmer que c'est le français, langue de ma mère, la première que j'ai entendue. Or, étant née au Brésil, dans un foyer fréquenté quotidiennement par des Brésiliens, le portugais est entré dans ma vie simultanément.

Jusqu'à l'âge de cinq ans, lorsque j'ai commencé à aller à l'école (brésilienne), le français a certainement été la langue la plus présente dans ma vie. Langue 


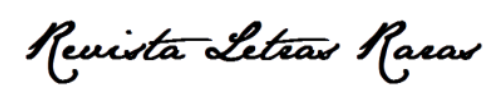

ISSN: 2317-2347 - v. 9, Dossier Spécial (2020)

Todo o conteúdo da RLR está licenciado sob Creative Commons Atribuição 4.0 Internacional

parlée avec mes parents, langue de la tendresse mais également des remontrances, langue de la prière nocturne. Le souvenir le plus marquant du français est sans doute celui des lectures faites par ma mère, au moment d'aller au lit : le ton réconfortant de sa voix, le bruissement des pages tournées et toujours la même déception au moment où l'histoire s'interrompait...

J'ai été entourée depuis toujours par le portugais. Mon père était pasteur et tout son travail se faisait en portugais. Mes parents s'adressaient donc à tous dans cette langue. Ce n'est qu'avec nous (les enfants) qu'ils parlaient en français. Je ne me souviens pas d'avoir " appris » le portugais. J'ai grandi au sein de ces deux langues. (COSTA, 2018, pp. 28, 29) ${ }^{4}$

Pourquoi tant de conflits? Parce que, comme l'affirme Revuz, "[t]oute tentative pour apprendre une autre langue vient déranger, interroger, modifier ce qui s'est inscrit en nous avec les mots de cette langue première [la langue dite maternelle] » (REVUZ, 1991, p.26).

Cela veut dire que l'apprentissage d'une autre langue ébranle aussi nos répertoires et nos représentations culturelles car le nouveau système linguistique appris est beaucoup plus qu'un système : langue et culture forment un binôme inséparable, aucun mot n'est neutre ou une simple étiquette accolée à une chose, à un concept. L'exemple typique, très connu des Brésiliens, est l'incapacité de traduire le mot « saudade » en français. II faut donc que l'apprenant développe une capacité d'ouverture vers un autre système de références et de valeurs, courant le risque, encore une fois de la perte, de l'exil.

3 De l'imitation à la création, le passage par l'autre pour devenir un autre dans une classe de FLE au Brésil

Comment l'apprentissage d'une nouvelle langue, avec tout ce que cela implique, a-t-il vraiment lieu?

\footnotetext{
${ }^{4}$ Traduction de Béatrice Reichen V. Costa pour cet article. Texte original :

"A dificuldade começa com a escolha da língua... Devo escrever em português ou em francês? Qual é minha língua materna? Qual é minha primeira língua?

Se eu me referir ao sentido literal da expressão 'língua materna', posso dizer que é o francês, língua de minha mãe, a primeira que ouvi. No entanto, tendo nascido no Brasil e em uma casa frequentada diariamente por brasileiros, 0 português entrou em minha vida simultaneamente.

Até a idade de cinco anos, quando comecei a frequentar a escola (brasileira), o francês certamente foi a língua mais presente em minha vida. Língua falada com meus pais, língua do carinho, mas também dos sermões e correções, língua da prece noturna. Talvez a lembrança mais marcante do francês seja a das leituras feitas por minha mãe na hora de dormir: o tom de voz reconfortante, o farfalhar das páginas viradas e sempre a mesma decepção quando a história era interrompida...

Fui rodeada desde sempre pelo português. Papai era pastor e todo seu trabalho acontecia em português. Meus pais, portanto, dirigiam-se a todos nessa língua, e só conosco falavam em francês. Não me lembro de ter 'aprendido' 0 português. Cresci em meio aos dois idiomas." (COSTA, 2018, pp. 28, 29)
} 


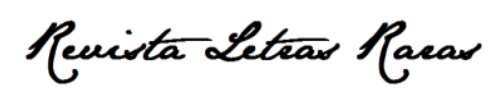

ISSN: 2317-2347 - v. 9, Dossier Spécial (2020)

Todo o conteúdo da RLR está licenciado sob Creative Commons Atribuição 4.0 Internacional

Les enseignants chercheurs dans le domaine de la didactique des langues sont toujours en quête de moments où il leur serait possible de voir le processus d'apprentissage en plein déroulement, comme un chirurgien qui voit battre le cœur de son patient au moment d'une intervention. De nombreux travaux y ont été consacrés, ayant comme base des apprenants qui sont parfois accompagnés pendant des années, comme ceux du programme ESF 5 .

Les écrivains qui ont choisi d'écrire dans une langue étrangère sont, nous l'avons vu, des sources importantes pour ce sujet. Laura Alcoba, qui a écrit Le bleu des abeilles, tout comme Mizubayashi, auteur d'Une langue venue d'ailleurs, sont tous deux bilingues. Leur apprentissage du français a des différences considérables, mais aussi des points en commun : Alcoba, née en Argentine, a dû aller à la rencontre de sa mère exilée en France à l'âge de dix ans et, pour cela, a suivi des leçons privées de français avant de partir :

Avec Noémie, j'ai découvert des sons nouveaux, un $r$ très humide que l'on va chercher tout au fond du palais, presque dans la gorge, et des voyelles qu'on laisse résonner sous le nez, comme si on voulait à la fois les prononcer et les garder un peu pour soi. Le français est une drôle de langue, elle lâche les sons et les retient en même temps, comme si, au fond, elle n'était pas tout à fait sûre de bien vouloir les laisser filer - je me souviens que c'est la première chose que je me suis dite. Et qu'il allait me falloir beaucoup d'entraînement, aussi. (ALCOBA, 2013, p. 12. En italique dans le texte)

Mizubayashi a décidé de commencer à apprendre la langue française tout seul à Tokyo:

C'est ainsi qu'en avril 1970, lorsque j'accédai enfin à l'université, le français entra dans ma vie, et commença à occuper tout mon quotidien. J'avais dix-huit ans et sept mois. Et je savais que le français allait m'accompagner pour toujours.

Mon premier contact avec cette langue s'est réalisé, je l'ai dit, à travers la leçon de français assurée à la Radio nationale par un professeur japonais en collaboration avec les deux invités dont j'ai souligné l'attrait vocal. J'écoutai toutes les leçons pendant deux ou trois semaines consécutives. Très vite, je sentis avec une sorte de douleur que tous les sons, tous les mots français effectivement entendus par mes oreilles dans leur matérialité sonore, étaient sans retour, définitivement perdus dans leur vibration éphémère ; je voulais les voir près de moi, comme on aime avoir à sa portée les $C D$ qu'on préfère, toujours prêts à être insérés dans un lecteur. (MIZUBAYASHI, 2011, p. 33)

\footnotetext{
5 Programme Acquisition des langues étrangères par des adultes immigrés, de la Fondation Européenne de Science (ESF), réalisé en cinq pays de 1982 à 1987. Selon Jorge Giacobbe (1989, pp. 69, 70), «le but général du programme était, pour chaque équipe qui y participait, de suivre dans son déroulement, à partir du moment d'arrivée, et pendant trois années, l'acquisition de la langue du pays d'accueil par des informateurs immigrés. Ces informateurs étaient de jeunes adultes des deux sexes, d'un niveau de scolarisation peu élevé. Les informateurs de l'équipe de Paris étaient des réfugiés politiques latino-américains.».
} 


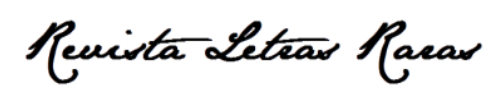

ISSN: 2317-2347 - v. 9, Dossier Spécial (2020)

Todo o conteúdo da RLR está licenciado sob Creative Commons Atribuição 4.0 Internacional

Le contexte d'apprentissage dans un milieu où les personnes engagées se comprennent dans la même langue maternelle peut paraître très sécurisant. Pourtant, il représente, lui aussi, un défi de taille pour ces apprenants, comme nous le verrons par la suite, car il y a toujours des transformations importantes provoquées par une nouvelle langue au niveau même de leur identité, parfois de vrais bouleversements tout à fait nécessaires pour une bonne maîtrise de ce nouveau monde parlant.

Dans son livre Identity and Language Learning Extending the Conversation, 2ème édition (2013), Bonny Norton part d'une étude longitudinale sur des femmes migrantes au Canada pour développer, entre autres, ses concepts d'identité et d'investissement dans le domaine de l'apprentissage des langues étrangères. Dans cette édition de son livre, l'auteure sud-africaine, Enseignante en Colombie britannique, pose deux questions qui me semblent fondamentales aussi pour le contexte auquel je fais référence dans cet article : sous quelles conditions les apprenants parlent, écoutent, lisent et écrivent ? Comment les relations de pouvoir sont-elles impliquées dans les négociations d'identité ? Pour Norton, l'engagement des apprenants dans les conversations en langue étrangère, essentiel à l'appropriation de cette langue, met en question non seulement le pouvoir mais aussi la reconnaissance sociale. Elle affirme :

Si les apprenants ne font pas de progrès dans leur apprentissage d'une deuxième langue, les enseignants ne peuvent pas en conclure qu'ils ne souhaitent pas apprendre cette langue ou qu'ils sont démotivés ou inflexibles ; peut-être sont-ils en pleine lutte pour y arriver parce qu'ils ne peuvent pas parler dans des conditions de marginalisation. (NORTON, 2013, p. 26) ${ }^{6}$

La classe de langue étrangère est, pour moi, un espace où les conditions de marginalisation sont très présentes, d'une autre façon, mais jouant, elles aussi, un rôle majeur, surtout quand il s'agit d'un apprentissage fait à l'âge adulte ou presque. Pour ce qui est du pouvoir, le contexte d'enseignement universitaire est très marqué, malheureusement, par une hiérarchie difficile à rompre. Parfois, même quand l'enseignant désire opérer cette rupture, certains étudiants ne l'acceptent pas et préfèrent la situation connue et rassurante de contrôle du professeur à l'inconnu déstabilisant du manque de contrôle et de hiérarchie, situation dans laquelle c'est à eux d'agir et de prendre des responsabilités, comme l'apprentissage en autonomie, si fondamentale dans ce contexte. Selon Claire Kramsch, dans l'introduction de la traduction de Norton (2013):

\footnotetext{
${ }^{6}$ Ma traduction. Texte original: "If learners do not make progress in learning, teachers cannot assume that learners do not wish to learn the second language or that they are unmotivated or inflexible; perhaps the learners are struggling because they cannot speak under conditions of marginalization."
} 


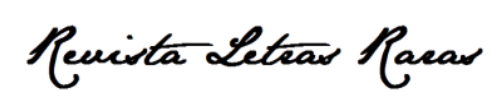

ISSN: 2317-2347 - v. 9, Dossier Spécial (2020)

Todo o conteúdo da RLR está licenciado sob Creative Commons Atribuição 4.0 Internacional

\begin{abstract}
l'agentivité des apprenants de langue contribue également à leur permettre de reconfigurer leurs relations à autrui et d'affirmer d'autres identités, plus puissantes, à partir desquelles parler, écrire ou lire, et donc à renforcer l'acquisition de la langue. (KRAMSCH apud ZEITER ; BEMPORAD, 2016, p. 190)
\end{abstract}

La condition asymétrique de pouvoir d'une classe de langue vient aussi du fait que l'enseignant, déjà investi du pouvoir induit par sa position hiérarchique, l'est également de celui qui lui est accordé par la maîtrise de la langue enseignée, c'est-à-dire un pouvoir discursif, et c'est dans les échanges, dans les interactions discursives, que les identités et les subjectivités sont créées, recréées, modifiées et contestées : elles sont négociées.

Un des facteurs de marginalisation, de manque de pouvoir, qui est en jeu dans la salle de classe de LE, est, à mon avis, la sensation d'appartenance à la langue étrangère. Moi-même, ayant commencé à étudier le français à l'université, j'ai mis longtemps à accepter que cette langue puisse m'appartenir ou que je pouvais lui appartenir. II me semblait absolument nécessaire de la maîtriser presque parfaitement pour pouvoir la parler avec l'autorité d'un « propriétaire ». Or, ce n'est qu'en parlant et en faisant toujours des erreurs que l'on s'approprie une langue, pour bien la maîtriser, éventuellement, un jour. Aujourd'hui, après avoir travaillé plus de trente d'ans comme enseignante de FLE au Brésil, je soutiens que les langues appartiennent à ceux qui les parlent, peu importe leur niveau de maîtrise et, que pour bien s'exprimer dans une langue, il faut aimer le faire, ce qui n'est possible qu'avec l'expression de la subjectivité. Toutefois, cela devient pénible, voire impossible, dans un contexte marqué par des limites, des contraintes et des enjeux de pouvoir.

Pour aggraver cette situation, l'expression orale, surtout celle des apprenants débutants, devient quelque chose de très ardu car, lors de l'apprentissage d'une nouvelle langue, ils se voient non seulement destitués de leur pouvoir linguistique, ce qui est déjà très inhibiteur en soi, mais ils connaissent en outre une régression très gênante. Selon Revuz (1991),

Commencer l'étude d'une langue étrangère, c'est se placer dans une situation de non-savoir absolu, c'est retourner au stade de l'infans, du bébé qui ne parle pas encore, (re)faire l'expérience de l'impuissance à se faire entendre. (Revuz, 1991, p.28)

Mizubayashi décrit très bien cette situation, qu'il illustre en citant Arimasa Mori, philosophe et essayiste japonais dont les textes écrits en français l'on enchanté: 


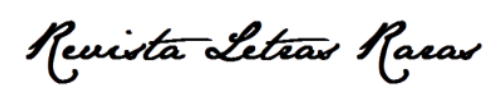

ISSN: 2317-2347 - v. 9, Dossier Spécial (2020)

Todo o conteúdo da RLR está licenciado sob Creative Commons Atribuição 4.0 Internacional

[...] il prit le risque de refaire sa vie, de renaître à une langue qui n'était pas la sienne et à la culture qui en est indissociable aussi bien qu'à la société des individus dont les rapports sont sans nul doute et largement déterminés par cette langue même. [...] Devant l'exigence de la langue française qui lui apparaît comme sollicitant une descente au fond des choses, une entière immersion de la part de celui qui désire l'apprendre, Mori accepte, chose incroyable après quarante ans d'apprentissage, de se reconnaître en la figure sidérante d'un jeune enfant (presque infans) qui arrive au monde et dans le monde, qui va naître tout juste à la langue et dans la langue. (MIZUBAYASHI, 2011, pp. 31, 32. En italique dans le texte original)

\section{De nouvelles perceptions au service des identités : les sens, les jeux et le théâtre en FLE}

Le grand défi de l'apprenant adulte, à mon avis, est de réussir à s'exprimer de manière subjective dans une situation d'apparente régression et de véritable asymétrie discursive. Dans ma pratique de classe, deux possibilités se sont montrées très porteuses pour cette expression.

La première consiste à faire travailler les étudiants sur les cinq sens ainsi que sur le sens de la mémoire ${ }^{7}$ afin de faire émerger chez eux ce qu'il y a de plus intime, de plus chargé d'émotions.

Ce travail part toujours d'un film et de textes littéraires sur l'un des sens. Ce premier contact avec un domaine peu exploré dans un contexte universitaire se fait donc par le moyen de l'art, dont l'importance est merveilleusement démontrée par John Dewey (Arte como experiência, 2010[1934]) et par Elliot Eisner (El arte y la creación de la mente, 2004). Une étude lexicale liée à des expériences sensorielles permet aux étudiants non seulement d'apprendre de nouveaux mots par la lecture et l'écoute, mais aussi par le goût », l' " odorat » et le " toucher » de ce nouveau lexique. En écrivant sur ce qu'ils ressentent pour une musique ou un arôme particulier, par exemple, ils se lancent sur la voie des expériences vécues dans leur enfance, parfois oubliées. Les résultats sont surprenants et toujours émouvants. On sait que la pratique d'une langue maternelle ou étrangère - sollicite le sujet, sa façon d'interagir avec les autres et avec le monde (Revuz, 1991), mais l'on ne peut découvrir l'autre que si l'on se découvre soi-même d'abord. Les étudiants qui participent à cette exposition aux sens se sont aussi découverts dans ce qu'ils ont de plus cher, de plus subjectif, et se sont montrés, par conséquent, plus ouverts au groupe, aux autres.

Les textes produits dans ces circonstances expriment bien le degré de subjectivité atteint et l'importance de cet aspect, ainsi que l'impact de la motivation et de l'affectivité (Bogaards,1991)

\footnotetext{
7 Jean-Yves et Marc Tadié (2004) considèrent la mémoire comme un sens à part entière.
}

\section{dol http://dx.doi.org/10.35572/rlr.v9i5.2010}




\section{Reuista Letras Parar}

ISSN: 2317-2347 - v. 9, Dossier Spécial (2020)

Todo o conteúdo da RLR está licenciado sob Creative Commons Atribuição 4.0 Internacional

pour l'apprentissage d'une langue étrangère. Les étudiants se rendent compte qu'ils sont vraiment considérés dans leur intégralité en salle de classe, ce qui est fondamental pour leur apprentissage ${ }^{8}$, et ce parcours défiant est lisible dans leurs productions textuelles. Ainsi, à partir de l'écoute du second mouvement de la gème symphonie d'Antonin Dvorak (la Symphonie du Nouveau Monde), B.C. écrit :

Je marche solitaire dans une forêt un peu obscure, par un chemin étroit, je pressens une impasse, quelque chose d'horrible ou de vraiment grandiloquent. Je suis solitaire dans un monde qui ne me connaît pas et solitaire je marche sous les branches des arbres. Des oiseaux qui ne me connaissent pas chantent et volent. Je pressens, cependant, une espèce de paix dans ce monde qui ne me connaît pas. Qu'est-ce que c'est?

II y a un sentiment dans moi-même qui me dit que ce chemin est certain... droit... il y a de bonnes nouvelles... Je ne sais pas pourquoi, mais il y a une impulsion qui doit me guider. Je me laisse aller, donc - un peu appréhensif, pourtant... Ce qui arrive sera peut-être triste, qui le saurait ?

[...] Maintenant je me sens plutôt sûr. Plutôt fort. Je marche plus vite, plus certain. Qui me donne ça ? Ça semble venir de la musique - l'exaltation sans but. [...] (B.C. Discipline de Français 6, 2010)

En ce qui concerne le travail avec l'odorat, deux exemples illustrent bien la prise en compte de la subjectivité et l'apprentissage d'un vocabulaire assez spécifique :

Pour moi, il ne suffit pas de lire, je dois sentir le papier, aspirer le doux arôme des pages d'une œuvre spéciale, humer le parfum des mots, des virgules et des points. (P.A. F5, 2012)

Pour moi, il n'existe pas d'odeur plus douce! Quand j'ai faim, froid ou peur, c'est son parfum que je cherche et qui me calme. Grâce à elle, mon bébé, je peux aspirer l'amour ! De Tout son corps et de son âme émane la tendresse, et ses cheveux exhalent une suave effluve. Elle aime aussi me flairer en me faisant sourire, et c'est pour ça que je l'aime tant ! (I.C., F5, 2012)

La deuxième voie que j'utilise pour faire émerger la subjectivité des apprenants est le travail avec les jeux de rôles et les mises en scène. Nancy Huston (1999) le dit admirablement:

Choisir à l'âge adulte, de son propre chef, de façon individuelle pour ne pas dire capricieuse, de quitter son pays et de conduire le reste de son existence dans une culture et une langue jusque-là étrangères, c'est accepter de s'installer à tout jamais dans l'imitation, le faire-semblant, le théâtre. (HUSTON, 1999, p. 30. En italique dans le texte).

\footnotetext{
${ }^{8}$ Comme l'affirme Patrick Anderson, « Le passage d'une langue à une autre est [...] un phénomène qui touche le sujet dans l'ensemble de son être ». (ANDERSON, 2015, p. 27)
} 


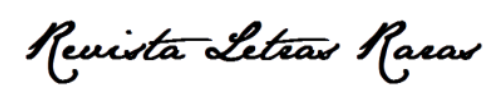

ISSN: 2317-2347 - v. 9, Dossier Spécial (2020)

Todo o conteúdo da RLR está licenciado sob Creative Commons Atribuição 4.0 Internacional

Faire de l'imitation et du théâtre une partie importante de l'apprentissage d'une nouvelle langue permet aux apprenants une construction plus solide car fondée sur la langue sentie, mise en bouche, expérimentée, comme l'affirme Maria da Glória Magalhães dos Reis dans $O$ texto teatral e o jogo dramático no ensino de Francês Língua Estrangeira (2008). Selon cette spécialiste, pour la réalisation d'une nouvelle énonciation dans la langue étrangère, la matérialité des mots, les sons et les rythmes nouveaux aident l'apprenant à se construire comme énonciateur, à acquérir une nouvelle voix.

Ce travail avec le théâtre, dans mes classes, part, lui-aussi de films, car le visionnement des gestes, des postures, des intonations typiques du français doit faire partie de l'apprentissage. II faut souligner que, dans la situation exolingue où ils se trouvent, les apprenants ont rarement l'occasion de « voir parler » des francophones, de les voir dans des situations pleines d'émotion, ce que le cinéma leur permet de faire. Les émotions, selon Georges Didi-Huberman (Quelle émotion! Quelle émotion?),

passent par les gestes que nous effectuons sans nous rendre compte qu'ils viennent de très loin dans le temps. Ces gestes [...] ont une très longue - et très inconsciente - histoire. Ils survivent en nous, même si nous sommes incapables de l'observer clairement sur nous-mêmes. (DIDI-HUBERMAN, 2013, pp. 39, 40. En italique dans le texte)

[...] une émotion, n'est-ce pas une é-motion, c'est-à-dire une motion, un mouvement, qui consiste à nous mettre hors de (é-, ex), hors de nous-mêmes ? Mais si l'émotion est un mouvement, elle est donc bien une action : quelque chose comme un geste tout à la fois extérieur et intérieur, puisque, quand l'émotion nous traverse, notre âme remue, tremble, s'agite, et notre corps fait des tas de choses dont nous n'avons même pas idée. (DIDI-HUBERMAN, 2013, pp. 30 à 32. En italique et entre guillemets dans le texte)

De cette manière, en 2019, pour aborder le sens de la mémoire et pour essayer de sensibiliser les apprenants, je leur ai montré le film La vie d'une autre, de Sylvie Testud (2012), avec Juliette Binoche et Mathieu Kassovitz, adapté du roman de Frédérique Deghelt (2007), avec bien des différences. Dans le livre, Marie, vingt-cinq ans, tombe follement amoureuse de Pablo un soir de fête. Après une nuit d'amour inoubliable, elle se réveille et constate que douze ans de sa vie se sont déjà passés, sans qu'elle en ait aucun souvenir... Dans le film, Marie a 41 ans et les dernières scènes de sa vie dont elle se souvient, lors de son réveil dans une chambre inconnue, ont eu lieu il y a 25 ans : la rencontre avec Paul, le coup de foudre, la nuit d'amour. Elle découvre aussitôt qu'elle a un enfant et une vie tout à fait opposée à celle qu'elle avait: 


\section{Revista Letear Pacar}

ISSN: 2317-2347 - v. 9, Dossier Spécial (2020)

Todo o conteúdo da RLR está licenciado sob Creative Commons Atribuição 4.0 Internacional

Dans une chambre, Marie se réveille, elle est étonnée.

Bruit d'aspirateur.

Marie: Paul ?

Rita: Non, madame, c'est moi, c'est Rita.

Bruit d'aspirateur.

Devant des photos :

Marie: C'est quoi cette connerie?

$[\ldots]$

Dans la chambre :

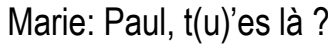

Devant le miroir :

Marie: Oh... Mes cheveux. Oh... C'est moi ? Oh... Paul, Paul, Paul, qu'est-ce qui se passe là? Je t'en prie.

$[\ldots]$

Avec Adam, l'enfant qu'elle découvre être son fils :

Marie: Écoute: c'est un jeu de réflexe. Tu (ne) réfléchis pas, tu réponds le plus vite possible. D'accord?

Adam: Papa dort avec toi ?

Marie: Non, c(e) (n)'est pas le jeu-là. Tu réponds vite sans réfléchir. Alors, ton année de naissance?

Adam: Euh...2000...

Marie: Ton nom, ton prénom?

Adam: Adam Speranski.

Marie: Ton adresse?

Adam: 7, rue Buenos Aires.

Marie: Et, pourquoi $t(u)(n)$ 'es pas à l'école?

Adam: Tu as oublié de m'emmener. Tu t'es réveillée trop tard.

Marie: Oui, c'est ça ! Et alors d'habitude tu y vas à quelle heure?

Adam: 8h45.

Marie: Et voilà. II est quelle heure-là?

Huit heures trente-quatre!

Adam: Pourquoi on (ne) prend pas l'ascenseur?

Marie: C'est le jeu ! Aujourd'hui tu guides. Tu fais les choses comme tu les ferais toi, hein ? C'est le jeu du «à l'envers».

Adam: Ouais !

Marie: Bon, dépêche-toi parce qu'on n'a plus que dix minutes.

On est à quel étage?

Adam: Le dernier.

Marie: Tu vois, c'est pourquoi je prends l'ascenseur d'habitude.

Comment s'appelle ton père ? Nom et prénom?

Adam: Paul Speranski.

Marie: Ta mère?

Adam: C'est toi.

Marie: Mais attends. C'est le jeu-là. Comment je m'appelle ? Nom et prénom ?

Adam: Marie Speranski.

Marie: Et ma mère, ta grand-mère de mon côté. Tu connais son prénom?

Adam: (réponse négative)

Marie: Où est ton père ? 


\title{
Reuista Letras Parar
}

ISSN: 2317-2347 - v. 9, Dossier Spécial (2020)

Todo o conteúdo da RLR está licenciado sob Creative Commons Atribuição 4.0 Internacional

\author{
Adam: À son atelier. \\ Marie: Qu'est-ce qu'il fait comme métier? \\ Adam: Bande Dessinée. \\ Marie: Et moi, je fais quoi? \\ Adam: Ben, du bureau, comme grand père. \\ Marie: J'ai quel âge? \\ Adam: 35 ans. \\ Marie: Tu es sûr? \\ Adam: Ah non, 41. T(u)'as 41 ans $^{9}$.
}

Après avoir vu le film et en avoir discuté, les étudiants ont transcrit les scènes ci-dessus, ce qui leur a permis de les réécouter une dizaine de fois. Puis, ils ont dû les rejouer en salle de classe.

II faut dire que, pendant ce premier semestre de 2019, j'ai eu le bonheur d'avoir avec moi une actrice, Giuliana Cerchiari, qui a proposé aux étudiants, dès la première séance de cours, des exercices de théâtre pour une plus grande conscience du corps et de soi. L'une des références théoriques et pratiques de Cerchiari est Moshé Feldenkrais (2014), physicien israélien d'origine russe qui a vécu en France et a mis au point une méthode de conscience corporelle à partir du mouvement et de son application dans l'image de soi, en faisant appel aux quatre parties intégrantes de l'action (le mouvement, la sensation, le sentiment, la pensée) dans une unité de fonctionnement.

Les étudiants se sont organisés en groupes et ont présenté leur reconstruction de la scène étudiée. La diversité des propositions a été étonnante : dans un groupe, huit étudiants, partagés en paires, ont joué Marie et Adam, chaque paire disant une réplique, les unes après les autres. Dans un autre groupe, deux étudiants ont joué la scène du miroir, se regardant l'un l'autre, comme s'ils étaient eux-mêmes un miroir, faisant les mêmes gestes et les mêmes expressions :
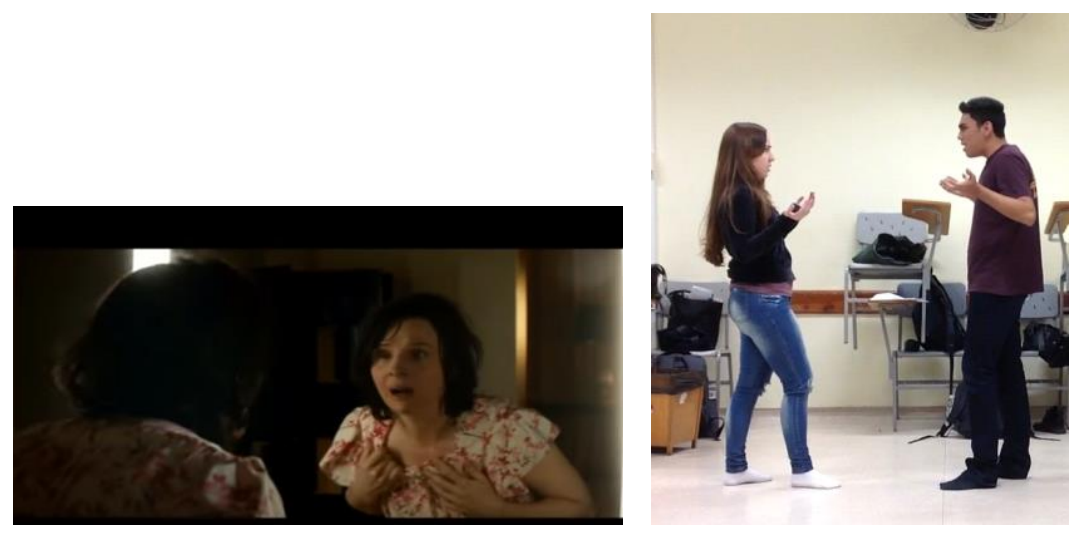

${ }^{9} \mathrm{Ce}$ dialogue peut remplacer parfaitement et de manière plus créative les célèbres fiches de présentation que les apprenants de FLE doivent remplir quand ils commencent à apprendre cette langue.

doi) http://dx.doi.org/10.35572/rlr.v9i5.2010 


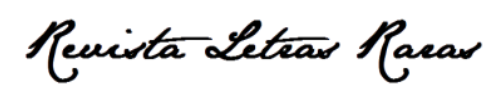

ISSN: 2317-2347 - v. 9, Dossier Spécial (2020)

Todo o conteúdo da RLR está licenciado sob Creative Commons Atribuição 4.0 Internacional

Cette mise en scène nous permet d'explorer l'importante notion d'effet de miroir qui a eu lieu : un garçon et une fille, Caroline et Vinícius, étudiants de français et parlant cette langue « sur scène », jouent Marie lors de son réveil dans le film et sa découverte d'une "nouvelle » Marie devant le miroir. Elle est maintenant une femme d'affaires réussie, insensible, une mère et une fille absente, tout à fait différente de ce qu'elle était à l'âge de 25 ans. Cette nouvelle Marie est, à son tour, tout autre dans le roman de Deghelt... Ce jeu de faux miroir presqu'infini nous rappelle un très beau chapitre du livre de Marina Yaguello, Alice au pays du langage (1981), « L'effet Vachequi-rit », que l'auteure illustre avec un passage d'Alice au Pays des Merveilles :

« N'imaginez jamais que vous ne soyez pas autre chose que ce qu'il pourrait sembler aux autres que vous étiez ou que ce que vous aviez été n'était pas autre chose que ce qu'il aurait semblé aux autres que vous auriez été autrement », dit la Duchesse à Alice. (CARROLL, 1865, apud YAGUELLO, 1981, p. 127)

Yaguello nous donne encore d'autres exemples de cet « emboîtement », dont le texte de Robert Desnos (2012[1953], p. 55) — « Maudit soit le père de l'épouse du forgeron qui forgea le fer de la cognée ... ") - et la pièce La cantatrice chauve, d'lonesco (1954), pour affirmer que la créativité et le désir de jeu entraînent des ruptures et des réinterprétations infinies pour les locuteurs. Afin de se sentir à l'aise dans une langue étrangère, les apprenants ont, eux aussi, besoin de jouer - et de s'amuser, d'avoir du plaisir, de s'émouvoir — dans et avec cette langue. Leur permettre de jouer, littéralement, dans la langue étrangère, c'est leur permettre de l'incorporer jusqu'à ce qu'elle devienne leur propre langue. C'est enfin les amener à se construire une nouvelle identité :

Comment s'étonner dès lors [...] que l'apprentissage d'une langue ne soit un processus essentiel et complexe ? Plus qu'une affaire de mémoire, on doit mobiliser son corps, son esprit, toute sa capacité de compréhension et d'imagination, puisqu'on apprend non un ensemble de mots et de règles, mais une manière de sentir, de percevoir, de raisonner, de déraisonner, de jurer, de prier et, finalement, d'être. (CHENG, 2002, pp. 9, 10).

En revendiquant l'importance du plaisir dans ce processus, et le plaisir de l'écoute, Anderson propose, à partir de sa lecture de l'œuvre de Mizubayashi (2011), la triade suivante: imitation, incorporation et appropriation : 


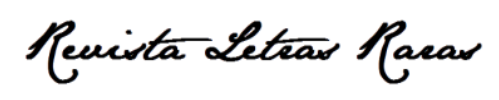

ISSN: 2317-2347 - v. 9, Dossier Spécial (2020)

Todo o conteúdo da RLR está licenciado sob Creative Commons Atribuição 4.0 Internacional

L'imitation est dans le sonore, c'est à la fois l'attirance pour devenir autre, mais également la séduction éprouvée par la voix de l'Autre.

[...] vient ensuite l'incorporation, elle prend différentes formes : c'est la relation à la musicalité, à la mélodie, à la voix parlée ou chantée, aux mots qui résonnent dans l'oreille, et dans la lecture qui passe également par le rapport sensuel de l'audition. [...]

C'est ensuite l'appropriation provoquée à la fois par l'incorporation des textes lus, parlés, dits, sus, et mémorisés. [...] [Elle] réside dans une incorporation de l'autre en soi qui fait que le sujet occupe une place vide et s'incorpore dans la langue. (ANDERSON, 2015, pp. 115 à 117).

Dans ce riche processus de la construction d'un nouveau « moi », d'une nouvelle voix, la confiance des apprenants envers leurs collègues et leurs enseignants est fondamentale, car les premiers se sentent très exposés, voire « nus », en salle de classe de langue étrangère. Plutôt que s'approprier une langue, il s'agit, comme je l'habitude de dire, de « se faire imprégner par l'autre langue ». Yves Érard, dans son article Trouver sa voix dans le paysage sonore d'une langue étrangère, écrit sur se laisser « traverser par la nouvelle langue »:

[...] mon expression dans une langue qui n'est pas la mienne ne consiste pas à m'approprier cette langue, mais à accepter de me laisser traverser par elle. L'expression de soi se tisse dans les expressions d'autrui. Je ne m'approprie donc pas une langue, c'est elle qui s'empare de moi. Puis-je supporter cette altération de mon expression par les mots des autres ? Cela dépendra de ma capacité à me rendre intelligible dans des rythmes et des musiques que je ne maîtrise pas encore. Cette intelligibilité sera fragile parce qu'elle impliquera toujours que je me reconnaisse moi et que je me fasse reconnaître dans un paysage sonore qui ne m'est pas encore bien familier. L'apprentissage d'une langue étrangère me demande un effort qui s'apparente à de la patience : visà-vis des autres, d'abord, vis-à-vis de moi-même, ensuite. Cette vision de la subjectivité dans une langue étrangère met en évidence que l'apprentissage d'une confiance en soi se construit symétriquement à l'apprentissage d'une confiance en autrui, d'une confiance en soi comme un autre. (ÉRARD, 2019, p. 203)

Le groupe d'étudiants qui a participé aux jeux de rôle et aux mises en scènes, pendant le troisième semestre de leur contact avec la langue française, en 2019, a relevé le défi mentionné par Érard : quelques-uns ont formé, le semestre d'après, une troupe de théâtre, Les plongeons, et se sont présentés avec la pièce L'été, de nouveau, de Laurent Van Wetter au 1er Festival de Théâtre Francophone au Brésil, réalisé pendant le XXIIème Congrès Brésiliens des Professeurs de Français à Brasília, 


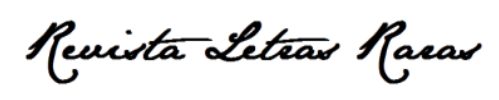

ISSN: 2317-2347 - v. 9, Dossier Spécial (2020)

Todo o conteúdo da RLR está licenciado sob Creative Commons Atribuição 4.0 Internacional

\section{Considérations finales}

Avec un apprentissage fait dans le plaisir, dans l'amour pour la langue étrangère, les apprenants améliorent leur expression orale et aussi, comme déjà présenté, leur expression écrite. Leurs textes, inouïs, sensibles, beaux, car subjectifs, présentent d'ailleurs toujours beaucoup moins d'erreurs que dans le résumé d'un texte scientifique (voilà un des mystères que j'aimerais expliquer un jour...). Pour cela, bien évidemment, il faut aussi que l'enseignant cherche des thèmes appropriés et incitateurs. Ainsi, le premier semestre de la présente année, 2020, après avoir travaillé avec des étudiants de la 2ème année de français sur le livre Dora Bruder, de Patrick Modiano (1997), qui part d'un avis de recherche, je leur ai demandé le travail suivant: « Vous devez rédiger un texte à partir d'un avis de recherche, vrai ou imaginé, sur une autre personne ou sur vous-même (pourquoi pas?). Ce n'est pas l'avis de recherche l'objet principal de ce texte, mais ce que vous écrivez à partir d'un tel déclencheur. Ajoutez-y des descriptions. »

Voici le texte d'une étudiante brésilienne, diplômée en droit et qui travaille comme assistante juridique dans un tribunal :

Petite fille de sept ans : aidez-moi à la retrouver S.V.P.

On recherche une fille âgée de sept ans, de taille moyenne (1m20), mince, les cheveux lisses et châtains avec une frange irrégulière probablement coupée par sa maman. À première vue, c'était la timidité de J. qui attirait l'attention, mais en regardant plus attentivement on verrait qu'elle avait toujours un visage ravissant avec des yeux bridés, marrons, étincelants et très curieux, ainsi qu'un sourire vif et franc comme celui des sœurs cadettes.

Si on la rencontrait dans la rue, on la verrait habillée de vêtements colorés et confortables - elle aimait surtout ses baskets lumineux qui la faisaient se promener dans la rue en regardant en arrière pour voir les petits éclats provenant de ses talons. Elle tiendrait probablement la main de ses parents Cécile et Albert et elle bavarderait sans arrêt, leur racontant ses dernières aventures à l'école et donnant de petits sauts en l'air.

$[\ldots]$

Cette petite fille J. est maintenant bien cachée et fluide sous les couches des années, des formations et des expériences de vie, formant un ensemble indescriptible. En cas d'information, c'est-à-dire d'une étincelle de cette fille de sept ans, veuillez le faire savoir à Juliana, 34 ans, puisqu'elle aimerait la revoir et rappeler les meilleurs souvenirs de son enfance même pour un instant fugace. (Juliana K.)

Peut-être Juliana pourra-t-elle rencontrer J. en français, langue étrangère qu'elle maîtrise de plus en plus et qui, surtout, lui permet de dire ce qu'elle ne dirait probablement pas en portugais... 


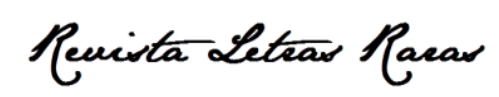

ISSN: 2317-2347 - v. 9, Dossier Spécial (2020)

Todo o conteúdo da RLR está licenciado sob Creative Commons Atribuição 4.0 Internacional

\section{Références}

ABREU, Marcella dos Santos. Ensino-aprendizagem de língua francesa na escola brasileira: memória, visibilidade e resistência de corpos pedagógicos. Thèse de Doctorat, FFLCH-USP, 2019.

ALCOBA, Laura. Le bleu des abeilles. Paris : Gallimard, 2013.

ANDERSON, Patrick. Une lange à venir. Paris : L'Harmattan, 2015.

BAKHTINE, Mikhaïl (N. V. VOLOCHINOV). Le Marxisme et la philosophie du langage, essai d'application de la méthode sociologique en linguistique. Traduit du russe et présenté par Marina Yaguello. Préface de Roman Jakobson. Paris: Ed. de Minuit, 1977[1929].

BOGAARDS, Paul. Aptitude et affectivité dans l'apprentissage des langues étrangères, Paris: Hatier/Didier, 1991.

CATROUXO, Sandra. Hors-la-loi, Poèmes. São Paulo : LFEdições, 2019.

CHENG, François. Le dialogue. Paris : Éditions Desclée de Brouwer, 2002.

CIORAN, Emil. Aveux et anathèmes. Paris: Gallimard, 1987.

CIORAN, Emil. La tentation d'exister. Paris: Gallimard, 1956.

COSTA, Béatrice Reichen V. Itinerãças. São Paulo: Paulistana, 2018.

DABÈNE, Louise et al. Variations et rituels en classe de langue, Paris: Didier, 1990.

DEGHELT, Frédérique. La vie d'une autre. Arles/Paris : Actes Sud, 2007.

DELLATORRE, Sahsha K. W. Práticas de leitura nos manuais escolares de francês (1930-1960). Mémoire de Master 2, FFLCH-USP, 2012.

DEWEY, John. Arte como experiência, traduit de l'anglais par Vera Ribeiro. São Paulo: Martins Fontes, 2010[1934].

EISNER, Elliot. El arte y la creación de la mente, traduit de l'anglais par Genis Sánchez Barberán. Barcelona, Buenos Aires, México : Paidós, 2004.

ÉRARD, Yves. Trouver sa voix dans le paysage sonore d'une langue étrangère, in A contrario 2019/1 ( $n^{\circ} 28$ ), pp. 203 à 234. Mis en ligne sur Cairn.info le 11/02/2020. Consulté le 14 novembre 2020.

FELDENKRAIS, Moshe. La puissance du moi: Exploitez toutes les facultés de votre cerveau et de votre corps. Paris : Marabout, 2014.

FENOGLIO, Irène. L'intime étrangeté de la langue, 2006, Langage \& Inconscient, $\mathrm{n}^{\circ} 2$, éd. Lambert-Lucas, 2006. Disponible sur http://www.item.ens.fr/articles-en-ligne/lintime-etrangete-dela-langue. Consulté le 30 octobre 2020.

GIACOBBE, Jorge. Construction des mots et construction du sens: cognition et interaction dans l'acquisition du français par des adultes hispanophones, Thèse de Doctorat en Linguistique, Université Paris VII, 1989.

GREEN, Julien. Le langage et son double, traduit de l'anglais par Julien Green. Paris : Editions du Seuil, 1987.

HUSTON, Nancy. Nord perdu, suivi de Douze France. Arles/Paris : Actes Sud, 1999. 


\section{Revista Letears Racar}

ISSN: 2317-2347 - v. 9, Dossier Spécial (2020)

Todo o conteúdo da RLR está licenciado sob Creative Commons Atribuição 4.0 Internacional

LAVAL, Martine. RAHIMI, Atiq : L'entretien, Télérama 3071, 19 novembre 2008, pp. 21-24.

MADJIDI, Maryam. Marx et la poupée. Paris : J'ai lu, 2017.

MAGALHÃES DOS REIS, Maria da Glória. O texto teatral e o jogo dramático no ensino de Francês Língua Estrangeira. Thèse de Doctorat. FFLCH-USP, 2008.

MODIANO, Patrick. Dora Bruder. Paris: Gallimard, 1997.

MIZUBAYASHI, Akira. Une langue venue d'ailleurs. Paris : Gallimard, 2011.

REVUZ, Christine. La langue étrangère entre le désir d'un ailleurs et le risque de l'exil. Education Permanente, nº 107, juin 1991.

TADIÉ, Jean-Marc; TADIÉ, Yves. Le sens de la mémoire. Paris : Folio, 2004.

VAN WETTER, Laurent. L'été, de nouveau. In Recueil 10 sur 10 : pièces francophones à jouer et à lire (Tome 2), DramEdition, Późna (Pologne), 2016.

VYGOTSKY, Lev Semenovitch. A construção do pensamento e da linguagem. São Paulo: WMF Martins Fontes. 2ème édition, 2009, traduit du russe par Paulo Bezerra. Ed. Original: 1934.

YAGUELLO, Marina. Alice au pays du langage. Pour comprendre la linguistique. Paris : Seuil, 1981.

ZEITER, Anne-Christel, BEMPORAD, Chiara. Identité et investissement dans l'acquisition des langues. Une traduction de l'introduction d'ldentity and Language Learning de Bonny Norton (2013)», Lidil [En ligne], 54, 2016, mis en ligne sur http://journals.openedition.org/lidi//4119 le 01 janvier 2017, consulté le 5 octobre 2020. 